\title{
Application of Local Mean Decomposition in Marine Gravity Anomaly Data Processing
}

\author{
Jun YIN \\ Second Institute of Oceanography, State Oceanic Administration, Hangzhou, 310012, China \\ 352567309@qq.com
}

Keywords: Marine Gravity; Local Mean Decomposition; De-noising; Wavelet Transform

\begin{abstract}
Marine gravity anomaly data interfered with noise impact of the follow-up data processing and analysis. Hereby a de-noising method of marine gravity anomaly data by local mean decomposition (LMD) combined with wavelet transform was proposed. De-nosing of marine gravity anomaly data requires maximum retention of useful information and as far as possible to suppression of noise interference. The collected marine gravity anomaly data is decomposed into a series of production functions (PF) by LMD, and wavelet de-noising carries on each PF with different scale factor, which can suppress the noise and protect the useful information from loss at the same time. Experimental data processing results show that the proposed method is effective.
\end{abstract}

\section{Introduction}

The earth's gravity field is a physical field reflecting the physical distribution of the earth, which restricts the movement of any object in the earth [1]. The earth's gravity field is closely related to the development of space technology, and it is the basic physical field information of digital earth. The ocean area is about 3610 square kilometers, accounting for $71.8 \%$ of the earth's surface area. Therefore the research of marine gravity field is of great significance [2]. At present, the acquisition of marine gravity data mainly includes sea surface ship gravity measurement, Marine airborne gravity measurement and satellite altimetry inversion of marine gravity. Unlike terrestrial gravity data measurements, marine gravity data are relatively uniform. With the development of science and technology and the growth of demand, the accuracy requirement of marine gravity data is more and more high [3][4]. No matter what kind of marine gravity measurement is adopted, the marine gravity anomaly data is often subject to noise interference, therefore, de-nosing is essential for marine gravity anomaly data processing and analysis. Due to the high accuracy of data processing in marine gravity anomaly, traditional de-nosing methods often cannot meet the requirements. LMD is a time frequency processing method for nonstationary signal, which decomposes the signal into a series PF for time frequency analysis. Based on LMD, the marine gravity anomaly data can carry de-noising on PF. The noise in the marine gravity anomaly data can be decomposed into each PF and the distribution of the noise would be changed compared with the total noise information. In all kinds of de-noising methods, wavelet de-noising has the advantages that it can deal with nonstationary signal, and the de-noising result varies with the scale factor different. Therefore, the wavelet de-noising carried on each PF with different scale factor can improve the performance effectively. The marine gravity anomaly data often has non-stationary characteristics. Therefore, it is very suitable to deal with wavelet de-noising. According to the characteristics of marine gravity anomaly data, we propose a method based on LMD combined with wavelet de-noising. Finally, experimental data are used to demonstrate the effectiveness of the proposed method.

\section{LMD and Wavelet De-nosing}

Similar to the empirical mode decomposition (EMD) method [5], LMD is an adaptive signal decomposition method which decomposes a signal to a series of PFs needs no basis functions, each PF corresponding to a product of an envelope signal and a pure FM signal. The implement step of LMD can be summarized as follows 
Step1: Determine all the local extreme points of the signal $x(t)$, including the maxima and minima $n_{11}\left(k_{l}\right)(l=1,2, \mathrm{~L}, m)$, local amplitude function $m_{11}(t)$ and local mean function $a_{11}(t)$ can be computed by

$$
\begin{aligned}
& m_{11}(t)=\frac{n_{11}\left(k_{l}\right)+n_{11}\left(k_{l+1}\right)}{2} \\
& a_{11}(t)=\frac{\left|n_{11}\left(k_{l}\right)-n_{11}\left(k_{l+1}\right)\right|}{2}
\end{aligned}
$$

where $k_{l}=k_{1}, k_{2}, \mathrm{~L}, k_{M-1}, \quad t \in\left[k_{l}, k_{l+1}\right]$.

Step2: Adopt the sliding average for all the local mean function and local amplitude function for smoothing. Then subtract the smoothing local mean function $n: p(t)$ from the original signal $x(t)$, and we get the separation signal $h_{11}(t)$. Normalize $h_{11}(t)$ by smooth local amplitude function $Q_{19}(t)$ is given by

$$
\begin{aligned}
& h_{11}(t)=x(t)-r P_{19}(t) \\
& s_{11}(t)=h_{11}(t) / \theta_{19}(t)
\end{aligned}
$$

Step3: if $s_{11}(t)$ is not a pure FM signal in [-1,1], take $s_{11}(t)$ as the original signal and repeat step1 to step2 till the separation signal meet the condition. Instantaneous phase and instantaneous frequency can be determined by

$$
\begin{gathered}
\varphi_{1}(t)=\arccos \left(s_{11}(t)\right) \\
f_{1}(t)=d \varphi_{1}(t) / 2 \pi d t
\end{gathered}
$$

Step4: Use all the local amplitude function product to obtain instantaneous amplitude is given by

$$
a_{1}(t)=a_{11}(t) a_{12}(t) \mathrm{L} \quad a_{1 n}(t)=\prod_{q=1}^{n} a_{1 q}(t)
$$

The first PF is given by

$$
P F_{1}(t)=a_{1}(t) s_{1 n}(t)
$$

Step5: Here separate the first PF from the original signal as follow

$u_{1}(t)=x(t)-P F_{1}(t)$

Then let $u_{1}(t)$ is a new original signal and repeat step1 to step4 till all $u_{k}(t)$ meet the monotone function condition. Thus, the original signal can be expressed as the sum of PF components and a monotonic component

$$
x(t)=\sum_{p=1}^{k} P F_{p}(t)+u_{k}(t)
$$

From the decomposition of LMD can see that each PF is a scale of the signal $x(t)$, if there is impulse noise interference, the noise will extent to every PF according to the scale of LMD. Although the LMD results cannot meet the ideal performance, it is not important for the subsequent de-noising process. Wavelet de-noising carried on each PF rather than the marine gravity anomaly data, and then the different scale factor of wavelet de-nosing can be set to obtain ideal de-nosing performance.

Wavelet analysis in engineering signal processing and analysis has been widely used in signal analysis which is called "microscope". Wavelet de-noising is a most commonly used method in the engineering data processing, due to the random noise in general can be assumed as Gaussian noise, and the noise distribution is often in the high frequency range, which analysis of wavelet de-noising is very favorable. Wavelet transform can decompose the signal into a series of approximate components and detail components, According to the principle of wavelet Mallat decomposition algorithm, the detail component of wavelet transform is the output of the high pass filter, which corresponds to the high frequency components of the data. Through the processing of the wavelet detail component or the approximate components of the higher frequency components, the 
suppression of the random noise can be realized. There are some limitations for de-noising by using the traditional filtering method, that is, there is incompatible contradiction between the local characteristics of the protection signal and the noise suppression. As a kind of special window processing, wavelet analysis can be used to analyze the approximation and detail of the signal, and it is often a very effective method to select wavelet in de-noising. The principle of wavelet de-nosing is shown in Fig.1.

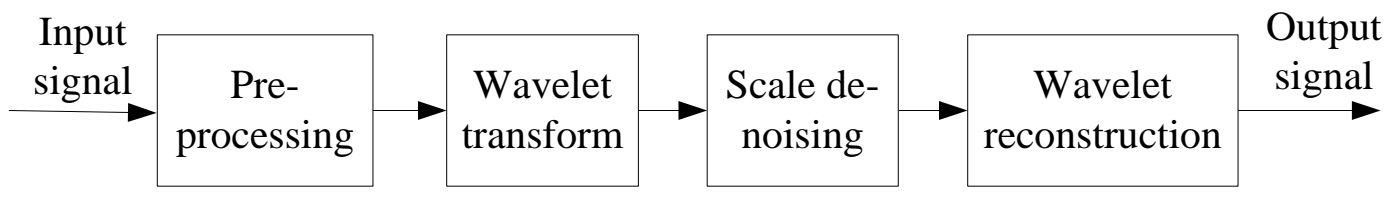

Fig. 1 The principle of wavelet de-nosing

Let $x(t)$ is the signal interfered with noise which is given by

$$
x(t)=s(t)+n(t)
$$

where $s(t)$ is the useful signal and $n(t)$ is the noise which meets the Gaussian distribution. $s(t)$ is usually low frequency signal and $n(t)$ is the high frequency signal. Therefore, wavelet de-noising can be carried on by following: Firstly, the signal is decomposed, and the noise signal contained in the high frequency details components, so the wavelet decomposition coefficient can be done by threshold processing and the signal is restored by wavelet reconstruction. The implement step of wavelet de-nosing can be summarized as follows

Step1: Wavelet decomposition of one dimensional signal. The signal is decomposed according to a suitable wavelet function and the number of decomposition levels, and then calculates the decomposition.

Step2: The high frequency coefficients of wavelet decomposition to perform threshold quantization operation. The quantitative processing is carried on the decomposition coefficients of high frequency according to a threshold.

Step3: One-dimensional wavelet reconstruction. The signal is reconstructed by wavelet reconstruction according to the high frequency coefficients of each layer, and then the signal after de-nosing is obtained.

The processing of high frequency detail coefficients generally has two methods. One is that the high frequency coefficients are all set to 0 , the other is that the high frequency coefficients process by the given threshold processing. Wavelet de-noising threshold functions have three types: default threshold function, soft threshold function and compelling threshold function. Let $n$ is the sum of the wavelet decomposition coefficients and $\sigma$ is the standard deviation of the noise, and the default threshold function can be given by

$$
t_{1}=\sigma \sqrt{2 \ln n}
$$

Let $t$ is the threshold value, the soft threshold function and the compelling threshold function can be given by Eq.13 and Eq.14 respectively

$$
\begin{aligned}
& y_{s}(x)=\left\{\begin{array}{cc}
\operatorname{sgn}(x)(|x|-t) & |x| \geq t \\
0 & |x|<t
\end{array}\right. \\
& y_{h}(x)= \begin{cases}x & |x| \geq t \\
0 & |x|<t\end{cases}
\end{aligned}
$$

\section{Wavelet De-nosing Combined with LMD}

According to the wavelet de-noising theory and process analysis, it can see that both de-nosing by setting the high frequency coefficients to 0 and adopting the wavelet threshold function, there are all related to the contradiction between the protection of useful information and the noise suppression. If the signal interfered with noise decomposes by LMD into series PF, the noise will be assigned to each PF, and then wavelet de-noising is carried on each PF with different scale factor 
for noise suppression to obtain better performance. The implementation process is shown in Fig.2.

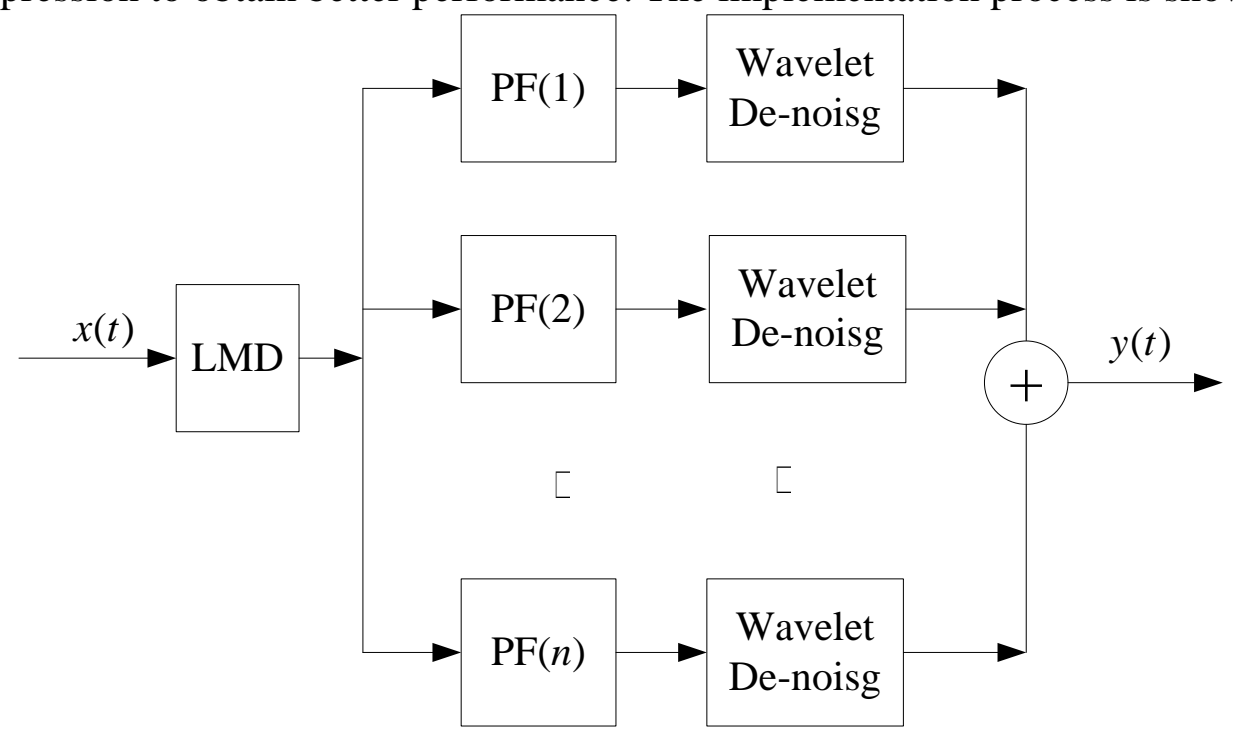

Fig.2 The diagram of wavelet de-noising combined with LMD

For LMD decomposes the signal according to the frequency distribution of the signal and the PF order from high frequency to low frequency. For the PF at higher frequency, wavelet de-noising can take the smaller scale factor. Otherwise, for the PF at lower frequency, wavelet de-noising can take the larger scale factor. This method can take advantage of wavelet de-nosing with different scale factors to suppress the noise and protect the useful information at the same time.

\section{Test results}

Marine gravity measurement is a fixed point or continuous measurement method at sea or seabed [6]. In marine surveying data processing, the measured data must calculate into the geoid to let the theoretical model fits the homogeneous ellipsoid hypothesis [7]. In the data process, it needs not only considering the error measurement system brought in under the special environment of the ocean, but also taking different treatment methods for different marine environment. However, here we only study the de-noising of the marine gravity data interfered with noise by the proposed method, which is the basis for a large number of subsequent processing. The marine gravity anomaly data of 15 measuring points in a sea area are processed and the 2-dimensional and 3-dimensional of the data are shown in Fig.3 and Fig.4 respectively. Due to noise interference, data interpretation becomes difficult. The processing results are shown in Fig.5 and Fig.6 respectively, it can be seen that the method proposed in this paper can effectively suppress the noise in the marine gravity anomaly data.

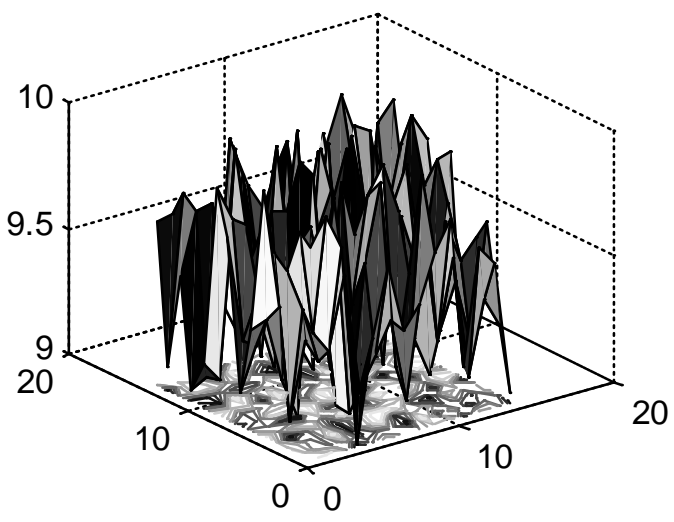

Fig.3 3-dimensional of data with noise

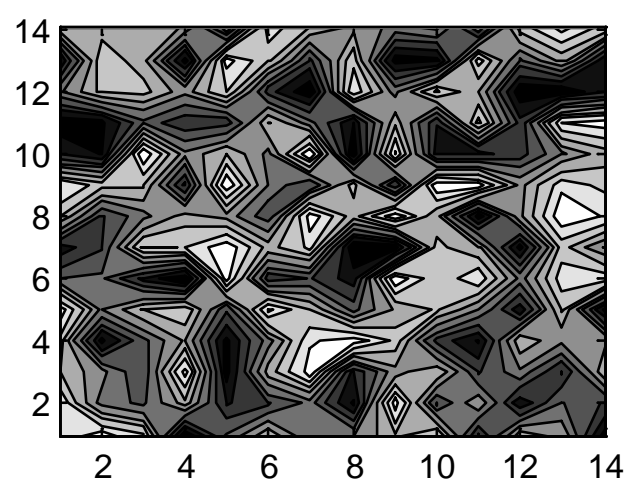

Fig.4 2-dimensional of data with noise 


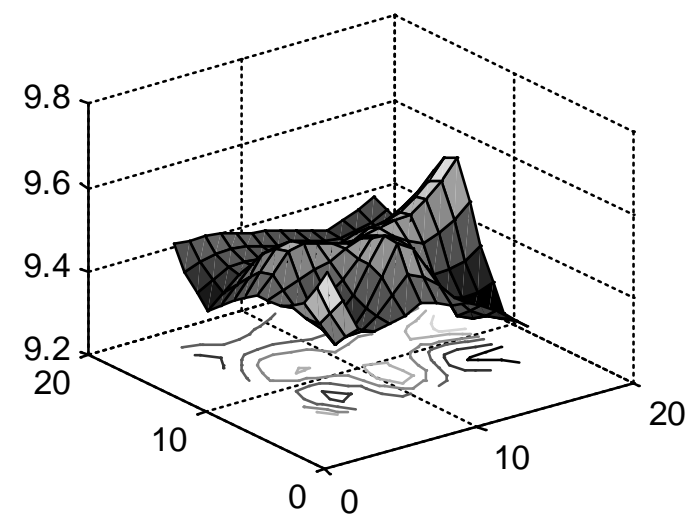

Fig. 5 3-dimensional of data after de-noising

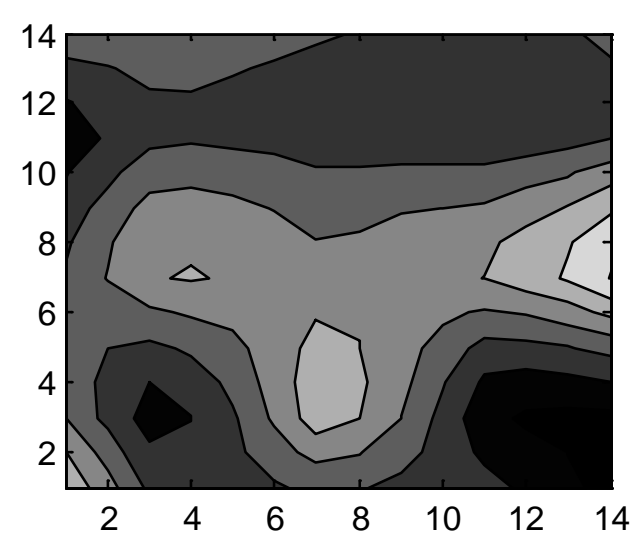

Fig.6 2-dimensional of data after de-nosing

\section{Conclusion}

This work proposed a de-noising method for marine gravity anomaly data by LMD combined with wavelet de-noising. The marine gravity anomaly data is decomposed into series PF by LMD before wavelet de-noising, thus wavelet de-noising can carried on PF with different scale factor rather than on the data directly. The proposed method can suppress the noise interference and protect the useful information at the same time, which has great significance for high precision processing of marine gravity anomaly data. The effectiveness of the method is proved by the results of data processing.

\section{References}

[1] WU D. Q., HUANG M. T., OUYANG Y. Z., et al. The study of high precision interpolation technology in marine gravity anomaly [J]. Science of Surveying and Mapping, 2008, 33(5): 70-72.

[2] LV C. C., HAO T. Y., XU Y. Complete bouguer correction in the oceanic gravity exploration [J]. Progress in Geophysics (in Chinese), 2009, 24(2): 513-521.

[3] NIE Z.X., WANG Z.J. The regulation method of the ground gravity downward continuation [J]. Progress in Geophysics (in Chinese), 2013, 28(6): 2872-2877.

[4] YANG C. B., LIU J. Y., WU Y. G., et al. Two-dimensional multi-scale discrete wavelet/wavelet packet analysis for models of regional and residual gravity anomaly separation [J]. Progress in Geophysics (in Chinese), 2010, 25(3): 1007-1014.

[5] ZHANG S.X., CHEN C., WANG L. S., et al. The bidimensional empirical mode decomposition and its application to denosing and separation of potential field [J]. Progress in Geophysics (in Chinese), 2015, 30(6): 2855-2862.

[6] HU L. S., WANG J. L., WU J. S. The application progress of the genetic algorithm in geophysics [J]. Progress in Geophysics (in Chinese), 2002, 17(4): 598-604.

[7] MO H. H., LIU J. D., YU B. B., et al. Gravity anomalies based on least square collocation and matlab [J]. Journal of Geomatics, 2015, 40(1): 25-28. 